\title{
Fermion field in the vicinity of a brane
}

\author{
Takashi Taneichi \\ Renko-ji, Tama-shi, Tokyo, Japan; sanshiro201@yahoo.co.jp \\ Received 24 July 2013; revised 24 August 2013; accepted 30 August 2013
}

Copyright (C) 2013 Takashi Taneichi. This is an open access article distributed under the Creative Commons Attribution License, which permits unrestricted use, distribution, and reproduction in any medium, provided the original work is properly cited.

\begin{abstract}
A mechanism that enables fermions to live outside of but be localized near a brane is proposed based on the field-theoretical framework. Two types of fermion fields are considered: one is localized to the brane through the Yukawa interaction, and another is localized through the fermion interaction. It is shown that, besides the well-localized zero-mode, there exists a light mode continuum of which oscillating components are delocalized. As a consequence it is possible to assume existence of invisible material particles that live outside the brane on which we reside and beleaguer visible particles. A possible extension to the mind-body problem is discussed.
\end{abstract}

Keywords: Brane World; Field Localization; Extra Dimension; Mind-Body Problem

\section{INTRODUCTION}

What kind of function of our brains can bring about our mind? Modern science can hardly present any convincing answer to this frequently asked question from ancient times. Progress of measurement technologies such as fMRI (functional magnetic resonance imaging) has made it possible to visualize the brain hemodynamics. Consequently the relation between states of consciousness such as feeling, sense, and thought, and activity states of brains is becoming clear. This does not mean, however, that any proof of brain activities producing mental states is obtained. What it gives us is a relevance or a relationship between them. Causal relation is not proved at any rate. Even if bloodstream in a brain stops, the brain stops its activity, and the brain waves become flat, it is not impossible to regain life unless brain cells perish. In addition, there may be the need to examine mind activity to exist independent of brain activity when we consider the example that a person could mention, after he/she regained his/her consciousness, his/her memory of what had happened in his/her neighborhood, or the feelings that had come up in his/her mind while his/her brain was stopping its activity [1-3]. Having said that, however, we cannot think the brain activity and the mind independent of substances which exist irrelatively with each other at all, concerning the example of above-mentioned fMRI. There may still be unknown schemes of connection with mental states inside our brain, although it may or may not be the origin of mind. If substances as the subjects that conduct the mind activities, sometimes called consciousness or personality, exist independently from our brains, and if they are the cause of those brain activities which are observed by above-mentioned visualization technology, then it will be called into question next about how the existence scheme of the substances and the mechanism of the interaction of them with the brains are elucidated. Furthermore, if we consider these substances as not spiritual but physical ones, it must be elucidated then what have prevented them from being observed so far by medical and/or biological means. Motivated by the present status of the brain science, in this study a new way of existence scheme of material particles is discussed, in which the dwelling scheme of a soul in a body persistently based on the materialism comes in sight. More concretely, dwell of a soul based on materials in a body (or a brain) based on materials, as well, is discussed in terms of the field theory. Such an idea may have some contribution to future efforts toward understanding the existence form of consciousness as an independent body from brains. I claim the consciousness outside the brains to be made of ordinary matter, not be spiritual nor psychic. Based on the viewpoints of multi-dimensional universe, body of the consciousness is assumed to consist of matter that resides beyond the extra dimensions. Dwelling of the body of consciousness as matter, in our brain as matter, as well, is modelled by the fermion interaction between matter particles.

Concept of the extra dimension was born in the pursuit of unifying the electro-weak and the gravitational interaction in 1920's by Kaluza and Klein. The vision of the universe, in which $(3+1)$ dimensional universe is em- 
bedded in a higher dimensional bulk space-time, shows a route to solve various problems of modern physics. These include the hierarchy problem of electro-weak and Planck [4-6], origin of the dark matter [7], and the cause of the cosmic expansion $[8,9]$. In the field theoretical description of the 3-brane, the braneworld in which we live is described as a topological defect in a higher dimensional bulk, and low dimensional standard model particle modes are trapped on it [10]. It is a problem need to be solved to figure out a plausible scenario that restrains particle motions to the low dimensional space-time sheet. D-brane model in the framework of the string theory, the restriction of particle motion is naturally obtained by the fact that the standard model particles are described by the open strings, and their terminal points move exclusively on the D-brane $[11,12]$. In the field theoretical model, on the other hand, mechanisms that localize low energy fourdimensional vector (gauge) fields [10,13-22], and the scalar and fermion fields [23-35] were proposed. In this study, another type of localization mechanism of the fermion field based on a field theoretical model is discussed. A scalar field which a defect arises from and a fermion field that is trapped on a brane are considered. This fermion is called the host fermion. Another fermion field is considered, which is localized on the brane via the existing host fermion field. This fermion is called the parasite fermion. The parasite fermions couple to the host fermions with the fermion interaction, feel the potential that is formed by the host fermion field, and are localized around the brane. Formulation that identifies structure and nature of the potential is given below.

\section{STRUCTURE OF THE MODEL}

The model considered in the present work follows the braneworld idea in which the brane is a topological defect in the 5D bulk, called domain-wall. The bulk is assumed to be flat, that is

$$
d s^{2}=g_{A B}=\eta_{\mu \nu} d x^{\mu} d x^{v}-d z^{2}
$$

$g_{A B}$ and $\eta_{\mu v}$ are the five-dimensional and the Minkowski four dimensional metric tensor, respectively. $A$ and $B$ run 0 through 4 , and $\mu$ and $v$, on the other hand, 0 through 3. $x^{\mu}$ are the four dimensional and $z$ is the fifth dimensional coordinates, respectively. The brane is assumed to be localized at the origin of $z$. The total lagrangian density at hand is

$$
L_{\text {total }}=L_{\Phi}+L_{\Psi}+L_{\text {int }},
$$

where

$$
\begin{gathered}
L_{\Phi}=\frac{1}{2} g^{A B} \partial_{A} \partial_{B} \Phi-V(\Phi), \\
L_{\Psi}=i \bar{\Psi}_{1} \Gamma^{A} \partial_{A} \Psi_{1}+i \bar{\Psi}_{2} \Gamma^{A} \partial_{A} \Psi_{2},
\end{gathered}
$$

and

$$
L_{\text {int }}=-\kappa \bar{\Psi}_{2} \bar{\Psi}_{1} \Phi \Psi_{1} \Psi_{2}-\lambda \bar{\Psi}_{1} \Phi \Psi_{1},
$$

for $\Gamma^{\mu}=\gamma^{\mu}$, where $\gamma^{\mu}$ are the Dirac matrices. Domain-wall solution can be obtained from the equation of motion for real scalar field $\Phi$ derived from the lagrangian. The potential, $V(\Phi)$, has the form that gives the (anti-)kink solution

$$
V(\Phi)=\frac{x}{4}\left(\Phi^{2}-\mu^{2}\right)^{2},
$$

where $\chi$ and $\eta$ are constants of the potential. Two types of matter fields are considered. $\Psi_{1}$ is a fermion field that couples to the scalar field $\Phi$ through the Yukawa interaction of which the coupling constant is $\lambda$. $\Psi_{2}$ is a fermion field that interacts with $\Psi_{1}$ through the fermion coupling term whose coupling constant is $\kappa$, and is localized around the brane indirectly through the fermion coupling. $\Psi_{1}$ is the "host fermion" which visible matters around us are all concerned with. All fermions that have been observed experimentally in the past are in this category. $\Psi_{2}$, on the other hand, is what I call "parasite fermion" and the parasite fermion field can be localized around the brane only when it couples to the host fermion field through the fermion coupling. From the lagrangean density one can obtain an equation of motion for $\Psi_{2}$ such that

$$
i \gamma^{\mu} \partial_{\mu} \Psi_{2}+\gamma^{5} \partial_{Z} \Psi_{2}-\kappa \bar{\Psi}_{1} \Phi \Psi_{1} \Psi_{2} .
$$

Here $\Gamma^{4}=-i \gamma^{5}$ was used. Using the left- and righthanded 4D spinors $\gamma^{5} \Psi_{2 \mathrm{~L} / \mathrm{R}}=\mp \Psi_{2 \mathrm{~L} / \mathrm{R}} \Psi_{2 \mathrm{~L} / \mathrm{R}}$ that satisfy, the field is expanded, combined with the separating variable method, as

$$
\Psi_{2}(x, z)=f_{2 \mathrm{~L}}(z) \Psi_{2 \mathrm{~L}}(x)+f_{2 \mathrm{R}}(z) \Psi_{2 \mathrm{R}}(x) .
$$

$x$ are the four-dimensional coordinates. Substituting Equation (8) into Equation (7) leads to

$$
m_{2} f_{2 \mathrm{~L}}+\partial_{Z} f_{2 \mathrm{R}}-\kappa \bar{\Psi}_{1} \Phi \Psi_{1} f_{2 \mathrm{R}}=0,
$$

and

$$
m_{2} f_{2 \mathrm{R}}+\partial_{Z} f_{2 \mathrm{~L}}-\kappa \bar{\Psi}_{1} \Phi \Psi_{1} f_{2 \mathrm{~L}}=0 .
$$

$m_{2}$ is the apparent 4D mass of the trapped fermion such that

$$
i \gamma^{\mu} \partial_{\mu} \Psi_{2 \mathrm{~L} / \mathrm{R}}=m_{2} \Psi_{2 \mathrm{R} / \mathrm{L}} \text {. }
$$

Equations (9a) and (9b) lead to

$$
-\partial_{z z} f_{2 \mathrm{~L}}+W_{\mathrm{L}} f_{2 \mathrm{~L}}=m_{2}^{2} f_{2 \mathrm{~L}}
$$

and

$$
-\partial_{z z} f_{2 \mathrm{R}}+W_{\mathrm{R}} f_{2 \mathrm{R}}=m_{2}^{2} f_{2 \mathrm{R}}
$$

where

$$
W_{\mathrm{L}}=\kappa\left[\kappa\left(\bar{\Psi}_{1} \Phi \Psi_{1}\right)^{2}-\partial_{Z}\left(\bar{\Psi}_{1} \Phi \Psi_{1}\right)\right]
$$


and

$$
W_{\mathrm{R}}=\kappa\left[\kappa\left(\bar{\Psi}_{1} \Phi \Psi_{1}\right)^{2}+\partial_{Z}\left(\bar{\Psi}_{1} \Phi \Psi_{1}\right)\right] .
$$

$\partial_{z z}$ is the second order differential with respect to $z$. Stationary states of $f_{2 \mathrm{~L} / \mathrm{R}}$ obtained from Equations (11a) and (11b) shows the localization profiles of the left- and right-handed fermion fields that are defined by the potentials, $W_{\mathrm{L} / \mathrm{R}}$. Analytical solutions for $\Psi_{1}$ are used (for instance, see [35]). In case of $m_{1}=\frac{1}{\xi} \sqrt{2 \varepsilon-1}$, where $m_{1}$ is the effective mass of the trapped host fermion $\Psi_{1}$, $\xi$, the thickness of the domain wall defined by the potential (6) such that $\xi=1 /(\eta \sqrt{\chi / 2})$ and is set to the unit of $z$-coordinate, and $\varepsilon=\lambda \eta \xi$, the profiles of $W_{\mathrm{L}}$ is shown with a solid line in Figure 1. Presumable profile of $\left|f_{2 \mathrm{~L}}\right|^{2}$ is shown schematically with dotted line in the figure. Four $x$-dependent ( $z$-independent) terms that appear through $\bar{\Psi}_{1 \Theta} \Psi_{1 \Theta^{\prime}}\left(\Theta\right.$ and $\Theta^{\prime}=\mathrm{L}$ or R $)$ are assumed to be equal each other. We can see that the effective potentials asymptote to zero, implying that the $m_{2}$ zero mode is localized in the vicinity of the brane. There are also light modes that are oscillatory at infinity of $z$. This implies that at least one mode of $\Psi_{2}, m_{2}$ zero mode, is localized distantly from the brane, in contrast to the host fermions that are localized on the brane. Difference of the existence scheme results in the difference of how we, the residents of the brane, can access to the fermions. The host fermions and the parasite ones interact each other through the fermion interaction, but its strength depends on the extent of how the parasite fermions are localized, that is, their distances from the brane and the width of the probability density $\left|f_{2 \mathrm{~L}}\right|^{2}$.

\section{DISCUSSION}

Concerning the theory discussed in the previous section, it is shown that bound states of parasite fermions can exist, without assuming the existence of another brane,

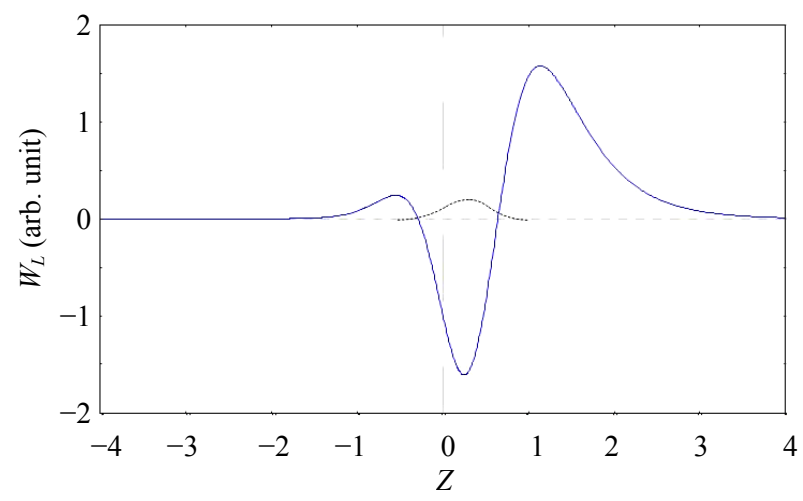

Figure 1. Potential $W_{\mathrm{L}}$ as a function of $z$, the extra-dimensional coordinate. that is apart from our brane in the extra-dimensional direction. The bound states seemingly function as if they result from fermion fields bound to another brane. Visibility of the bound states depends on how the bound fermion is apart from our brane, if we assume that it is impossible for photons to propagate across the bulk. Oscillating components of continuum of light modes, on the other hand, make it possible for residents of the brane to see this, however far the stable point of the potential lies against the brane. Fermions and gauge bosons that dwell in our brane can interact with the parasite fermions through its interaction with the oscillating components of the non-zero modes or the overlap of the zero mode to the brane, thus it is plausible to assume, at least theoretically, that we can see the parasite fermions if they exist. This type of existence scheme of fermions can present a possibility of a substantial modification in conventional viewpoints of some branches of science, such as the mindbody problem. Materialism reduces the existence scheme of mind into an assemblage of the chemical and biological actions inside the brains. Modern scientists who are willing to explain the mechanism of manifestation of mind search the origin of mind for the intra-brain phenomena such as neuronal firing. The best proof of this idea would be the manifestation of thought, sense, or emotion within an artificially prepared apparatus that realizes the neuronal firing inside it. Why? Reproductivity must be generally secured in natural science. Such apparatus, of course, has not been realized up to now.

Researchers in question, involved in neuroscience, confess honestly that the mechanism with which images appear and then disappear in the brains is purely made of material, and with which minds dwell in the brains is left entirely mysterious. They claim, on the one hand, that the neuronal firing is doubtlessly the cause by which our minds come into existence, on the other hand, however, they confess that their searches for the connection between the cause and the mind as its result are just about to get underway. There are, of course, researchers who search for mechanism other than the neural network. We can say, taking all in all, that the researches for the origin of the mind that have been undergone so far have not elucidated anything about the origin of the mind anyway. I may say that it is too early to conclude the source of mind is in the activity of neural network, since the connection between neuronal activity and manifestation of our mind is unidentified, although it is the core of this issue, and no one can even say, furthermore, that the circumstantial evidence is complete. Concept of the parasite fermion possibly releases us from the restriction of searching the origin of mind manifestation in the neural network and the brain at hand. If one can say that the natural scientific study of mind is about to get underway, then we must not exclude any type of possibility on the way 
to study and elucidation of the mind problem. Non-conventional type of multi-dimensional existence scheme of matter particles would serve as one of such possibilities.

\section{REFERENCES}

[1] Parnia, S. (2007) Do reports of consciousness during cardiac arrest hold the key to discovering the nature of consciousness? Medical Hypotheses, 69, 933-937. http://dx.doi.org/10.1016/j.mehy.2007.01.076

[2] Parnia, S. and Fenwick, P. (2002) Near death experiences in cardiac arrest: Visions of a dying brain or visions of a new science of consciousness. Resuscitation, 52, 5-11. http://dx.doi.org/10.1016/S0300-9572(01)00469-5

[3] Parnia, S., Walker, D., Yeates, R. and Fenwick, P. (2001) A qualitative and quantitative study of the incidence, features and aetiology of near death experiences in cardiac arrest survivors. Resuscitation, 48, 149-156. http://dx.doi.org/10.1016/S0300-9572(00)00328-2

[4] Arkani-Hamed, N., Dimopoulos, S. and March-Russell, J. (2001) Stabilization of submillimeter dimensions: The new guise of the hierarchy problem. Physical Review D, 63, 064020 .

http://dx.doi.org/10.1103/PhysRevD.63.064020

[5] Randall, L. and Sundrum, R. (1999) Large mass hierarchy from a small extra dimension. Physical Review Letters, 83, 3370-3373.

http://dx.doi.org/10.1103/PhysRevLett.83.3370

[6] Arkani-Hamed, N., Hall, L., Smith, D. and Weiner, N. (2000) Solving the hierarchy problem with exponentially large dimensions. Physical Review D, 62, 105002. http://dx.doi.org/10.1103/PhysRevD.62.105002

[7] Arkani-Hamed, N., Dimopoulos, S., Dvali, G. and Kaloper, N. (2000) Manyfold universe. Journal of High Energy Physics, 12, 010.

[8] Khoury, J., Ovrut, B.A., Steinhardt, P.J. and Turok, N. (2001) Ekpyrotic universe: Colliding branes and the origin of the hot big bang. Physical Review D, 64, 123522. http://dx.doi.org/10.1103/PhysRevD.64.123522

[9] Rasanen, S. (2002) On ekpyrotic brane collisions. $\mathrm{Nu}$ clear Physics B, 626, 183-206. http://dx.doi.org/10.1016/S0550-3213(02)00056-1

[10] Arkani-Hamed, N., Dimopoulos, S. and Dvali, G. (1998) The hierarchy problem and new dimensions at a millimeter. Physics Letters B, 429, 263-272. http://dx.doi.org/10.1016/S0370-2693(98)00466-3

[11] Horava, P. and Witten, E. (1996) Heterotic and Type I string dynamics from eleven dimensions. Nuclear Physics $B, \mathbf{4 6 0}, 506-524$. http://dx.doi.org/10.1016/0550-3213(95)00621-4

[12] Lukas, A., Ovrut, B.A., Stelle, K.S. and Waldram, D. (1999) Universe as a domain wall. Physical Review D, 59, 086001. http://dx.doi.org/10.1103/PhysRevD.59.086001

[13] Dvali, G. and Shifman, M. (1997) Domain walls in strongly coupled theories. Physics Letters B, 396, 64-69. http://dx.doi.org/10.1016/S0370-2693(97)00131-7

[14] Dvali, G., Gabadadze, G. and Shifman, M. (2000) (Quasi)
Localized gauge field on a brane: Dissipating cosmic radiation to extra dimensions?

[15] Dimopoulos, P., Farakos, K., Kehagias, A. and Koutsoumbas, G. (2000) Lattice evidence for gauge field localization on a brane.

[16] Duff, M.J., Liu, J.T. and Sabra, W.A. (2001) Localization of supergravity on the brane. Nuclear Physics B, 605, 234-244.

http://dx.doi.org/10.1016/S0550-3213(01)00160-2

[17] Ghoroku, K. and Nakamura, A. (2002) Massive vector trapping as a gauge boson on a brane. Physical Review D, 65, 084017.

http://dx.doi.org/10.1103/PhysRevD.65.084017

[18] Akhmedov, E.Kh. (2001) Dynamical localization of gauge fields on a brane. Physics Letters B, 521, 79-86. http://dx.doi.org/10.1016/S0370-2693(01)01176-5

[19] Dvali, G., Gabadadze, G. and Porrati, M. (2000) 4D gravity on a brane in 5D Minkowski space. Physics Letters $B$, 485, 208-214. http://dx.doi.org/10.1016/S0370-2693(00)00669-9

[20] Dubovsky, S.L. and Rubakov, V.A. (2001) On models of gauge field localization on a brane. International Journal of Modern Physics A, 16, 4331-4349. http://dx.doi.org/10.1142/S0217751X01005286

[21] Liu, Y.-X., Zhao, Z.-H., Wei, S.-W. and Duan, Y.-S. (2009) Bulk matters on symmetric and asymmetric de Sitter thick branes. Journal of Cosmology and Astroparticle Physics, 2, 3.

[22] Liu, Y.-X., Zhang, L.-D., Wei, S.-W. and Duan, Y.-S. (2008) Localization and mass spectrum of matters on Weyl thick branes. Journal of High Energy Physics, 8, 41.

[23] Bajc, B. and Gabadadze, G. (2000) Localization of matter and cosmological constant on a brane in anti de Sitter space. Physics Letters B, 474, 282-291. http://dx.doi.org/10.1016/S0370-2693(00)00055-1

[24] Randjbar-Daemi, S. and Shaposhnikov, M. (2001) Fermion zero-modes on brane-worlds.

[25] Akama, K. (2000) An early proposal of "brane world".

[26] Rubakov, V.A. and Shaposhnikov, M.E. (1983) Do we live inside a domain wall? Physics Letters B, 125, 136-138. http://dx.doi.org/10.1016/0370-2693(83)91253-4

[27] Pavsic, M. (1986) Einstein's gravity from a first order lagrangian in an embedding space. Physics Letters A, 116, 1-5. http://dx.doi.org/10.1016/0375-9601(86)90344-0

[28] Bryan, R. (1999) Are the Dirac particles of the Standard Model dynamically confined states in a higher dimensional flat space? Canadian Journal of Physics, 77, 197-210. http://dx.doi.org/10.1139/p99-012

[29] Gregory, R., Rubakov, V.A. and Sibiryakov, S.M. (2000) Brane worlds: The gravity of escaping matter. Classical and Quantum Gravity, 17, 4437-4450. http://dx.doi.org/10.1088/0264-9381/17/21/306

[30] Dubovsky, S.L., Rubakov, V.A. and Tinyakov, P.G. (2000) Brane world: Disappearing massive matter. Physical Review $D, \mathbf{6 2}, 105011$. http://dx.doi.org/10.1103/PhysRevD.62.105011

[31] Pavsic, M. (2001) A brane world model with intersecting 
branes. Physics Letters A, 283, 8-14.

http://dx.doi.org/10.1016/S0375-9601(01)00189-X

[32] Kehagias, A. and Tamvakis, K. (2001) Localized gravitons, gauge bosons and chiral fermions in smooth spaces generated by a bounce. Physics Letters B, 504, 38-46. http://dx.doi.org/10.1016/S0370-2693(01)00274-X

[33] Ringeval, C., Peter, P. and Uzan, J.P. (2002) Localization of massive fermions on the brane. Physical Review D, 65, 044016. http://dx.doi.org/10.1103/PhysRevD.65.044016
[34] Melfo, A., Pantoja, N. and Tempo, J.D. (2006) Fermion localization on thick branes. Physical Review D, 73, 044033. http://dx.doi.org/10.1103/PhysRevD.73.044033

[35] Sarrazin, M. and Petit, F. (2010) Equivalence between domain-walls and "noncommutative" two-sheeted spacetimes: Model-independent matter swapping between branes. 